CASE REPORT

\title{
Long-term Adverse Effects of Acute Myeloid Leukemia Treatment on Odontogenesis in a Child
}

\author{
Magali Hernandez ${ }^{1}$, Cécile Pochon ${ }^{2}$, Pascal Chastagner ${ }^{3}$, Dominique Droz ${ }^{4}$
}

\begin{abstract}
Background: Several studies showed that cancer therapies during tooth development are associated with dental abnormalities, including enamel defects, arrested tooth development, microdontic teeth, and agenesis.

Study design: We describe the case of a nine-year-old boy treated for acute myeloid leukemia at 15 months of age, who presents several dental abnormalities resulting from anticancer treatment.

Results: The patient was included and treated according to the ELAM 02 French protocol. Six years after allogenic hematopoietic stem cell transplantation, the intraoral and radiographic examination highlighted the agenesis of the second permanent molars and three of the four second premolars, microdontia of the first premolars, root stunting of the central incisors and first premolars, rootlessness of the first permanent molars, and enamel defects localized at the permanent incisors and canines. As a first step to reduce enamel defects, restorations with resin composite (Tetric EvoCeram ${ }^{\circledast}$ A2, Ivoclar Vivadent) were performed under a dental dam. Orthodontic treatment was contraindicated due to arrested tooth development, short roots, and a risk of resorption is considered too important.
\end{abstract}

Conclusion: The young age at diagnosis ( $<5$ years of age) and intensive chemotherapy (especially myeloablative conditioning with high doses of cyclophosphamide and Busulfan) could explain the severity of the dental abnormalities. This case illustrates the importance of systematically scheduling a dental follow-up in parallel with the onco-hematologic follow-up allowing the clinicians to prevent, detect, and propose early intervention for dental late effects.

Keywords: Childhood cancer, Long-term survivors, Tooth abnormalities.

International Journal of Clinical Pediatric Dentistry (2019): 10.5005/jp-journals-10005-1614

\section{INTRODUCTION}

Over the past decades, the survival rate for childhood cancer has increased substantially. This can be explained by more effective chemotherapy regimens. Improvement in survival has led to delayed emergence of sequelae in long-term survivors such as heart failure, infertility, or risk of secondary malignancy. Furthermore, several studies have shown an association between cancer treatments such as radiotherapy and/or chemotherapy and dental developmental abnormalities, including agenesis, root stunting, and enamel defects. Indeed, radiation interferes with the dental development by directly inhibiting the mitotic activity of odontoblasts. Although the risk of dental abnormalities after radiotherapy is well described, the factors associated with long-term dental effects after chemotherapy for childhood malignancies are less known. Chemotherapies received during dental development affect odontogenesis and amelogenesis in childhood malignancy survivors. These anomalies are described in children treated for solid tumors ${ }^{1}$ or hematological malignancies ${ }^{2}$ such as acute lymphoid leukemia. Although these anomalies represent half of childhood cancers, acute myeloid leukemia (AML) occurs only in 15 to $20 \%$ of acute leukemia cases ${ }^{3}$ and long-term side effects involving tooth development are not often reported. Here, we report the case of a nine-year-old boy treated for AML at 15 months of age, who presents several dental abnormalities resulting from anticancer treatments.

\section{Case Description}

A young patient was diagnosed with acute myeloid leukemia (AML) at 15 months of age. He was included and treated according to the ELAM 02 French protocol. This treatment protocol contains induction chemotherapy with aracytine and novantrone, each course lasting

\footnotetext{
1,4Pediatric Dentistry Department, Children Hospital, CHRU Nancy, France; Pediatric Dental Department, Faculty of Dentistry, Nancy, France 2,3 Hematology, Oncology Department, Children Hospital, CHRU Nancy, France

Corresponding Author: Magali Hernandez, Pediatric Dentistry Department, Children Hospital, CHRU Nancy, France; Pediatric Dental Department, Faculty of Dentistry, Nancy, France, Phone: +33 0383154880 , e-mail:magali.hernandez@univ-lorraine.fr

How to cite this article: Hernandez M, Pochon $C$, et al. Long-term Adverse Effects of Acute Myeloid Leukemia Treatment on Odontogenesis in a Child. Int J Clin Pediatr Dent 2019;12(3):243-246.

Source of support: Nil

Conflict of interest: None
}

7 days and 5 days, respectively. The first complete remission was obtained so that the induction chemotherapy was followed by three courses of consolidation chemotherapy. The patient received aracytine, amsacrine, daunorubicin, etoposide, and l-asparaginase. Six months after the end of chemotherapy, a bone-marrow relapse was diagnosed. The patient received a new induction chemotherapy with the FLAG scheme (fludarabine, high-dose aracytine, and G-CSF). Due to poor response, a second-line induction chemotherapy with the IDAFLAG scheme (idarubicin, fludarabine, and high-dose aracytine) was administrated to the patient, leading to a complete response. The doses of chemotherapeutic agents are listed in Table 1. Allogeneic hematopoietic stem cell transplantation (allo-HCT) was then performed after a myeloablative conditioning, including busulfan $(1.2 \mathrm{mg} / \mathrm{kg} / \times 4$ per day $\times 4$ days $)$ and cyclophosphamide $(50 \mathrm{mg} /$ $\mathrm{kg} /$ day $\times 4$ days). The patient received cyclosporine, methotrexate, 
Table 1: Doses of chemotherapeutic agents according to the patient received

\begin{tabular}{|c|c|c|}
\hline \multicolumn{3}{|c|}{ Induction chemotherapy } \\
\hline \multicolumn{2}{|l|}{ Aracytine } & $200 \mathrm{mg} / \mathrm{m}^{2} /$ day $\times 7$ days \\
\hline \multicolumn{2}{|l|}{ Novantrone } & $10 \mathrm{mg} / \mathrm{m}^{2} /$ day $\times 5$ days \\
\hline \multicolumn{3}{|c|}{ Consolidation chemotherapy } \\
\hline \multirow[t]{2}{*}{ No. 1} & Aracytine & $3 \mathrm{~g} / \mathrm{m}^{2} \times 2 /$ day $\times 3$ days \\
\hline & Amsacrine & $100 \mathrm{mg} / \mathrm{m}^{2} \times 2 /$ day $\times 3$ days \\
\hline \multirow[t]{3}{*}{ No. 2} & Aracytine & $200 \mathrm{mg} / \mathrm{m}^{2} /$ day $\times 4$ days \\
\hline & Daunorubicine & $40 \mathrm{mg} / \mathrm{m}^{2} /$ day $\times 4$ days \\
\hline & Etoposide & $100 \mathrm{mg} / \mathrm{m}^{2} /$ day $\times 4$ days \\
\hline \multirow[t]{2}{*}{ No. 3} & Aracytine & $3 \mathrm{~g} / \mathrm{m}^{2} \times 2 /$ day D1, D2, D8, D9 \\
\hline & L-asparaginase & $6000 \mathrm{U} / \mathrm{m}^{2} /$ day D2, D9 \\
\hline \multicolumn{3}{|l|}{ FLAG } \\
\hline & Fludarabine & $30 \mathrm{mg} / \mathrm{m}^{2} /$ day from D2 to D6 \\
\hline & Aracytine & $2 \mathrm{~g} / \mathrm{m}^{2} /$ day from D2 to D6 \\
\hline & G-CSF & From D1 to D6 \\
\hline \multicolumn{3}{|l|}{ IDAFLAG } \\
\hline & Idarubicin & $8 \mathrm{mg} / \mathrm{m}^{2} /$ day D2, D4, J6 \\
\hline & Fludarabine & $30 \mathrm{mg} / \mathrm{m}^{2} /$ day from D2 to D6 \\
\hline & Aracytine & $2 \mathrm{~g} / \mathrm{m}^{2} /$ day from D2 to D6 \\
\hline & G-CSF & From D1 to D6 \\
\hline
\end{tabular}

and anti-thymoglobulin for graft vs host disease (GvHD) prophylaxis. He developed grade II-acute gastrointestinal tract and skin GvHD treated with steroids and mycophenolate mofetil, and then extensive chronic GvHD (gut, skin, and lungs), which required multiple immunosuppressive drugs (mycophenolate mofetil, infliximab, rituximab, tacrolimus, basiliximab, and steroid therapy for 2 years) associated with extracorporeal photochemotherapy, antibiotic prophylaxis (amoxicillin), and anti-viral drug (zovirax). The patient also suffered from pulmonary aspergillosis and mucormycosis treated with antifungal therapy (amphotericin B, voriconazole, caspofungin).

Six years after allo-HCT, the patient was referred to the Department of Pediatric Dentistry. The intraoral examination revealed structural anomalies such as enamel defects located at the permanent maxillary and mandibular central and lateral incisors and canines (Fig. 1A).
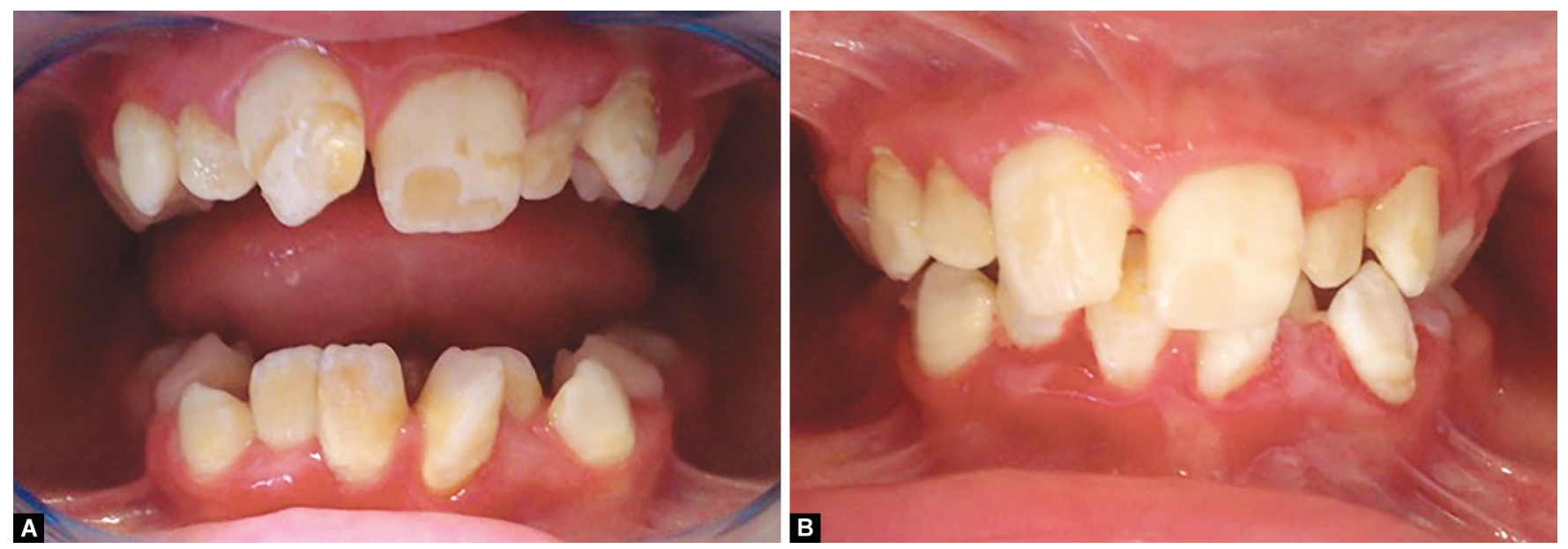

Figs $1 \mathrm{~A}$ and $\mathrm{B}$ : (A) Enamel defects located at the permanent incisors and canines in a nine-year-old patient treated for AML with chemotherapies and allo-HCT at 15 months of age; (B) Resin composite restorations (Tetric EvoCeram ${ }^{\circledR}$ A2, Ivoclar Vivadent) of the permanent maxillary incisors and canines 9 months after the restorations were performed molars and three second premolar (asterisks), microdontia of the first premolars (white arrows), root stunting of the maxillary and mandibular central incisors and first premolars (black arrows), and rootlessness of the first permanent molars (arrow heads) in a nine-year-old patient treated for AML

The dental panoramic radiograph (Fig. 2) showed several dental developmental abnormalities including agenesis of the second permanent molars and three second premolars, microdontia of the first premolars, root stunting of the maxillary and mandibular central incisors and first premolars, and rootlessness of the first permanent molars. Dental examination was completed by an orthodontic examination for advice regarding his malocclusion. Orthodontic treatment was contraindicated due to the arrested tooth development and short roots. The risk of root resorption was considered too important. As a first step to reduce enamel defects, we proposed to perform restorations with resin composite (Tetric EvoCeram ${ }^{\circledast} \mathrm{A} 2$, Ivoclar Vivadent) after micro-abrasion realized under a dental dam (Fig. 1B). The patient and his parents accepted only the restorations of the permanent maxillary incisors and canines. A systematic scheduling of a dental follow-up in parallel with the onco-hematologic follow-up was established. The objectives were (1) maintaining the primary teeth on the dental arch as long as 
possible (initiate a preventive treatment that contains application of fluorinated varnish and filling the grooves of the occlusal face for example, the purpose being to avoid the development of carious lesions), (2) observing the eruption of permanent teeth to detect structural anomalies, (3) evaluating the mobility of permanent teeth with short roots, (4) proposing a prosthetic treatment to replace missing teeth, prevent disorders in orofacial functions and promote the bone growth, and (5) meeting expectations of the patient and his parents. On the prosthetic plan, a removable prosthesis could be indicated and must be regularly adjusted to the bone growth. The bone remodeling process and the bone growth are considerable and do not allow the placement of implant during this period of growth. Implant therapies must be postponed and considered when the bone growth is complete.

\section{Discussion}

Proc et al. ${ }^{4}$ showed that dental abnormalities were most frequently observed in cancer survivors than in healthy patients (62\% vs $13 \%$, $p$ value $<0.0001)$. Cytotoxic molecules at the time of early dental development may result in agenesis, microdontia, and enamel defects, while effects of antineoplastic agents in the later stages of formation are characterized by the anomalies of root formation. ${ }^{5-7}$ Considering the sequence of dental calcification and development, ${ }^{8}$ children between 0 and 5 years of age seem to be at the highest risk of several dental abnormalities resulting from anticancer treatments. Indeed, several studies reported an age-specific risk for dental abnormalities. ${ }^{5,9-11}$ This explains the important number of missing teeth and the enamel defects observed in our patient, who received cancer treatment at 15 months of age.

It is given that chemotherapies include several molecules, and it is difficult to determine the independent effects of each antineoplastic agent on dental anomalies. However, previous studies have identified that cyclophosphamide and vincristine are associated with dental developmental disturbance in the survivors of childhood malignancies. ${ }^{1,9,10}$ Furthermore, several studies reported that children conditioned with total body irradiation (TBI) and high dose chemotherapy (cyclophosphamide and busulfan) before $\mathrm{HCT}$ are at high risk for dental developmental abnormalities. ${ }^{12,13}$ Considering this data, our patient, who received myeloablative doses of busulfan and cyclophosphamide for HCT, was at a high risk for long-term several dental abnormalities. His clinical picture confirms this hypothesis. It has also been suggested that the early use of antibiotics (amoxicillin) under the age of 4 years can disturb dental hard tissue formation and lead to enamel defects such as molar incisor hypomineralization $(\mathrm{MIH}){ }^{14}$ but the etiology of $\mathrm{MIH}$ is still unclear. In our patient, antibiotic prophylaxis (amoxicillin) was associated with the treatment of the GvHD from 2.5 to 8 years of age. To date, there has been no reported case of dental abnormalities such as agenesis, root stunting, microdontic teeth or enamel defects associated with immunosuppressive therapy in children. This case clearly illustrates the extent of oral late side effects of anticancer treatment in the survivors of childhood cancer and confirms that the severity of dental abnormalities is influenced by both younger ages at chemotherapy and by high doses of some antineoplastic agents and maybe some antibiotic and immunosuppressive therapies.

The impact of odontogenesis disturbance is also psychological. Dental anomalies in childhood cancer survivors are obviously not life-threatening complications, but they can impair the quality of life of teenagers and young adults. The occurrence of these dental anomalies is a reminder of cancer pathology, as they are a visible stigma. Dental anomalies decrease masticatory functions, maxillary bone growth, and may lead to orthodontic troubles but also impact the aesthetic view of the anterior region, especially enamel defects. A study showed that young people may make negative psychosocial judgments on the basis of enamel appearance..$^{15}$ Enamel defects should not be ignored and the practitioner should pay attention to the dental anomalies as far as the appearance of some enamel defects can be improved by simple non-invasive procedures such as micro-abrasion and composite restorations. ${ }^{16}$

More knowledge into the prevalence of these adverse late effects and risk factors associated is necessary to determine and prevent dental abnormalities to inform and provide support to the patient and his family.

\section{Clinical Significance}

A twice yearly dental examination carried out by a dental surgeon experienced in survivorship care, as recommended by the Children's Oncology Group guidelines, ${ }^{17}$ should be a part of medical follow-up monitoring late effects in long-term survivors of childhood cancer. This case illustrates the importance of systematically scheduling a dental follow-up in parallel with the onco-hematologic follow-up allowing the clinicians to prevent, detect, and propose early intervention of dental late effects.

\section{References}

1. Marec-Berard P, Azzi D, et al. Long-term effects of chemotherapy on dental status in children treated for nephroblastoma. Pediatr Hematol Oncol 2005;22:581-588. DOI: 10.1080/08880010500198848.

2. Maciel JCC, De Castro CG, et al. Oral health and dental anomalies in patients treated for leukemia in childhood and adolescence. Pediatr Blood Cancer 2009;53:361-365. DOI: 10.1002/pbc.22108.

3. Preudhomme C, Llopis L, et al. Classification et facteurs pronostiques des leucémies aiguës. Encycl Med Chir Hematol 2012;17(1):1-17. DOI: 10.1016/S1155-1984(12)55654-2.

4. Proc P, Szczepanska J, et al. Dental anomalies as late adverse effect among young children treated for cancer. Cancer Res Treat 2016;48(2):658-667. DOI: 10.4143/crt.2015.193.

5. Gawade PL, Hudson MM, et al. A systematic review of dental late effects in survivors of childhood cancer. Pediatr Blood Cancer 2014;61:407-416. DOI: 10.1002/pbc.24842.

6. Effinger KE, Migliorati CA, et al. Oral and dental late effects in survivors of childhood cancer: a children's oncology group report. Support Care Cancer 2014;22:2009-2019. DOI: 10.1007/s00520-014-2260-x.

7. Van der Pas-van Voskuilen IGM, Veerkamp JSJ, et al. Long-term adverse effects of hematopoietic stem cell transplantation on dental development in children. Support Care Cancer 2009;17:1169-1175. DOI: 10.1007/s00520-008-0567-1.

8. Schour I, Massler M. Studies in tooth development - the growth patterns of human teeth. Part 1 and 2. Amer Dent Ass 1940;27: 1778-1739. DOI: 10.14219/jada.archive.1940.0340.

9. Hsieh SG, Hibbert S, et al. Association of cyclophosphamide use with dental developmental defects and salivary gland dysfunction in recipients of childhood antineoplastic therapy. Cancer 2011;117: 2219-2227. DOI: 10.1002/cncr.25704.

10. Kaste SC, Goodman P, et al. Impact of radiation and chemotherapy on risk of dental abnormalities: a report from the childhood cancer survivor study. Cancer 2009;115:5817-5827. DOI: 10.1002/cncr.24670.

11. Wilberg P, Kannellopoulos A, et al. Dental abnormalities after chemotherapy in long-term survivors of childhood acute lymphoblastic leukemia 7-40 years after diagnosis. Support Care Cancer 2016;24:1497-1506. DOI: 10.1007/s00520-015-2940-1.

12. Vesterbacka M, Ringden $O$, et al. Disturbances in dental development and craniofacial growth in children treated with hematopoietic 
AML: Dental Late Effects

stem cell transplantation. Orthod Craniofac Res 2012;15:21-29. DOI: 10.1111/j.1601-6343.2011.01533.x.

13. Holtta $\mathrm{P}$, Alaluusua $\mathrm{S}$, et al. Agenesis and microdontia of permanent teeth as late adverse effects after stem cell transplantation in young children. Cancer 2005;103:181-190. DOI: 10.1002/ cncr.20762.

14. Laisi S, Ess A, et al. Amoxicillin may cause molar incisor hypomineralization. J Dent Res 2009;88(2):132-136. DOI: 10.1177/0022034508328334.
15. Craig SA, Baker SR, et al. How do children view other children who have visible enamel defects? Int J Paediatr Dent 2015;25:399-408. DOI: 10.1111/ipd.12146.

16. Nixon PJ, Robinson S, et al. Conservative aesthetic techniques for discolored teeth: 2 microabrasion and composite. Dent Update 2007;34:160-162. DOI: 10.12968/denu.2007.34.3.160.

17. Children's Oncology Group. Dental health following children cancer treatment. 2013. Available at: http://survivorshipguidelines.org/pdf/ healthlinks/English/dental_health_Eng.pdf., accessed: 2016-12-10. 